\title{
CRECIMIENTO DE LA MACHA Mesodesma donacium Y SU RELACIÓN CON EL FITOPLANCTON EN LA PLAYA POZO DE LIZAS (ILO, MOQUEGUA), DURANTE 1996-1997
}

Luis Rivera Chipana ${ }^{\prime}$ Olga Gómez Caballero ${ }^{2}$

\section{RESUMEN}

En el estudio del comportamiento del grupo de moluscos -especificamente del recurso macha Mesodesma donacium-para poder determinar su crecimiento, se tuvo en cuenta las cuatro estaciones durante los años 1996 y 1997, así como la relación con el fitoplancton.

\section{A B S TRACT}

In the study of the behavior of the group of mollusks specifically of the male resource Mesodesma donacium in order to determine their rate of growth, one kept in mind the four stations during the years 1996 and 1997, as well as the relationship with the phitoplankton.

\section{INTRODUCCIÓN}

Los mariscos en general tienen gran importancia como alimento, especialmente el recurso macha (Mesodesma donacium) para la población de la zona costera de la Provincia de llo.

Siendo muy importante contar con una información sistemática y constante para obtener datos en series de tiempo, se ha continuado con el estudio del bivalvo macha y conocer su estructura poblacional en períodos normales y anormales.

Este trabajo presenta el resultado de las muestras analizadas enloreferentealoscambios de distribución de este bivalvo.

El estudio se realizó en la Playa Pozo de Lizas, ubicada al sur de Punta Coles $\left(17^{\circ} 43^{\prime}\right.$ L.S. y $72^{\circ} 20^{\prime}$ L.W.) (Fig. 1).

\section{MATERIAL Y MÉTODOS}

Las muestras de agua de mar, para los estudios de fitoplancton, se tomaron mensualmente de enero de 1996 a diciembre de 1997, las que fueron fijadas con formalina neutralizada con Bicarbonato de Sodio. Asimismo, se obtuvieron registros de temperatura superficial del agua.

Para el análisis cuantitativo del fitoplancton se utilizó la metodología de Uterhmöl modificado, empleando cilindros de sedimentación de $50 \mathrm{ml}$. de capacidad. Los resultados se expresan en $\mathrm{N}^{\circ}$ cel/ 50 $\mathrm{ml}$.

Para el estudio del recurso macha se colectaron muestras mensualmente a partir de junio de 1996. Y en laboratorio se tomaron los datos biométricos (longitud total, altura, etc.). 


\section{RESULTADOS Y DISCUSIÓN}

\section{ASPECTOS FÍSICOS}

\section{Condiciones ambientales del mar}

Los registros de la temperatura superficial del agua de mar en Pozo de Lizas variaron entre 14.6 y 23.3 ${ }^{\circ} \mathrm{C}$., presentándose el valor más alto en el mes de diciembre de 1997 y el más bajo durante el mes de agosto de 1996, (Fig. 2).

En las temperaturas registradas en relación con la Temperatura Patrón, se aprecia que la mayor anomalia negativa se presentó en el mes de agosto de 1996 y fue de $-1.2^{\circ} \mathrm{C}$ y la positiva en el mes de diciembre de 1997, siendo de $+6.1^{\circ} \mathrm{C}$, ( Fig. 3).

\section{ASPECTOS BIOLÓGICOS}

\section{Composición y Densidad del Fitoplanctor}

En el área de estudio y durante todo el período de muestreo se observó una variación temporal en la composición y abundancia del fitoplancton.

Se han identificado 92 especies: 62 Diatomeas, 27 Dinoflagelados, 2 Silicoflagelados y/ 1 Fitoflagelado.

L.as Diatomeas fueron las más abundantes (Fig. 4), alcanzando el $99.5 \%$ ciel total de fitoplancton en 1996, las que estuvieron repiesentadas por: Denotula pumila, Guinardia delicatula y Asterionellopsis glacialis, especies pequeñas de alta tasa de reproducción; mientras que en el año de 1997, por la presencia de un aguaje en el mes de junio, los Dinoflagelados fueron los más abundantes, alcanzando el $98 \%$ del total de fitoplancton, la que estuvo representada principalmente por Gymnodinium splendens.

Asímismo se observó una variación temporal de las especies, durante 1996, notándose que en el verano dominaron Actinoptychus senarius $y$ Pleurosigma nicobaricum, en otoño predominaron los Chaetoceros. El invierno fué la estación más productiva. Además de las especies predominantes dominaron: Leptocylindrus mediterraneus, Th. subtilis y Proboscia indica. Y en primavera las más abundantes fueron: Cahetoceros radicans, Eucampia zoodiacus y Thalassiosira mendiolana.

En el año de 1997 las Diatomeas estuvieron pobremente representadas debido a la presencia de aguas calientes que se presentaron muy cerca de la costa; tal es asi que en la estación de verano predominó la Diatomea Proboscia indica y los Dinoflagelados: Ceratium furca y $C$. fusus fusus. En otoño predominaron los Dinoflagelados Gymnodinium splendens y Prorocentrum micans y las Diatomeas: Lauderia annulata, L. mediterraneus; en invierno, $G$. delicatula, D. pumila, Ch. socialis, Sk. costatumy otras especies de Chaetoceros.

En primavera dominaron las especies: $G$. delicatula, Hemiaulus sinensis y Thalassionema nitzschioides.

En las figuras 5 y 6 , para efectos de comparación, se muestran la distribución total de Diatomeas y Dinoflagelados. El año de 1995 fue considerado como un año normal, el año 1996 como período frio ó anti Niña y 1997 como caliente ó Niño.

En general, la Fig. 7 presenta cómo es que las Diatomeas, durante el año de 1995, alcanzaron valores elevados. En el mes de julio presenta valores de $88213 \mathrm{cel} / 50 \mathrm{ml}$., estuvo representada por $P$. pungens ( $86100 \mathrm{cel} / 50 \mathrm{ml}$.). En mayo con $82195 \mathrm{cel} /$ $50 \mathrm{ml}$., representada por las especies D. pumila $(61$ $900 \mathrm{cel} / \mathrm{ml}$.) en disminución. En agosto de 1996 ocurre un ligero florecimiento (50 $906 \mathrm{cel} / 50 \mathrm{ml}$.) representado por $G$. delicatula (45 $800 \mathrm{cel} / 50 \mathrm{ml}$.), para luego alcanzar valores muy bajos en el año de 1997 por efecto de EI Niño. En cuanto a los Dinoflagelados, en esta época normalmente aumentan en número y especies, solo que no se manifiesta en las muestras de agua; pọr esta razón están pobremente representados, con excepción de junio de 1997 en la que hubo un aguaje con $225491 \mathrm{cel} / 50 \mathrm{ml}$. producida por G. splendens.

\section{Variación Estacional del Fitoplancton}

En la Fig. 8 se presenta la variación estacional del fitoplancton observándose que la mayor abundancia se presentó en la estación de invierno de 1996 y otoño de 1997. Mientras que en la Fig. 9 se presenta la distribución del indice de la diversidad de fitoplancton en la playa Pozo de Lizas, de enero de 1995 a diciembre de 1997.

\section{Recurso Macha}

\section{Aspectos Biométricos}

Durante el período de estudio se obtuvo un total de 2592 ejemplares, que se presentan estacionalmente. 
En otoño de 1996 (Fig. 7) se obtuvieron 105 especímenes, cuyos tamaños fluctuaron entre los 61 y $81 \mathrm{~mm}$ de longitud, determinándose una longitud promedio de $70.4 \mathrm{~mm}$ y una moda de $71 \mathrm{~mm}$. En 1997 se obtuvieron 387 ejemplares, los tamaños fluctuaron entre los 63 y $87 \mathrm{~mm}$ de longitud, determinándose una longitud promedio de $74.0 \mathrm{~mm}$ y una moda en $77 \mathrm{~mm}$ y un porcentaje de 34 y 20.2 \%respectivamente.

En invierno de 1996 (Fig.8) se obtuvieron 359 especimenes, cuyos tamaños fluctuaron entre los 59 y $85 \mathrm{~mm}$ de longitud, determinándose una longitud promedio de $73.4 \mathrm{~mm}$ y dos modas en 65 y $63 \mathrm{~mm}$. En 1997 con 404 ejemplares, los tamaños fluctuaron entre los 65 y $89 \mathrm{~mm}$ de longitud, determinándose una longitud promedio de $76.5 \mathrm{~mm}$ y una moda en 77 $\mathrm{mm}$ y un porcentaje de 18.1 en ambos casos.

En primavera de 1996 (Fig. 9) se obtuvieron 436 especimenes, cuyos tamaños fluctuaron entre los 57 y $85 \mathrm{~mm}$ de longitud, determinándose una longitud promedio de $73.0 \mathrm{~mm}$ y una moda en $75 \mathrm{~mm}$. En 1997 con 414 ejemplares, !os tamaños fluctuaron entre los 59 y $89 \mathrm{~mm}$ de longitud determinándose una longitud promedic de $74.5 \mathrm{~mm}$ y una moda en $73 \mathrm{~mm}$ y un porcentaje de 18.8 y $16.4 \%$ respectivamente.

En verano de 1997 se obtuvieron 487 especímenes, cuyos tamaños fluctuaron entre los 57 y 89 $\mathrm{mm}$. de longitud, determinándose una longitud promedio di $7.1 .0 \mathrm{~mm}$ y una moda en $67 \mathrm{~mm}$ y un porcentaje de $19.9 \%$

En las playas arenosas la temperatura alcanza valores altos y el efecto de EI Niño se hace más notorio en las especies que habitan en la endofauna, tal es el caso de la macha que no soporta variaciones altas de temperatura. Durante el mes de diciembre de 1997 se observó una anomalia positiva de $+6.1^{\circ} \mathrm{C}$ la que persistió en el mes, de enero de 1998, causando la muerte y varazón de esta especie.

Asimismo cabe mencionar que con el fenómeno de El Niño se ha observado la presencia de Poliquetos en la valva superior del recurso macha, las mismas que pertenecen al Género Polidora sp. de la familia Spionidae, con esto se afirma que este fenómeno afecta positivamente para que el Poliqueto se reproduzca y se propague, ya que a medida que aumentaba la temperatura se incrementaba el número de ellos, encontrando en algunas valvas hasta 8 a 10 de estos poliquetos.

\section{CONCLUSIONES}

Al concluir el presente trabajo de investigación se ha podido arribar a las siguientes conclusiones:

- A partir del mes de abril de 1997 se pudo observar la presencia de aguas subtropicales superficiales registrando anomalias positivas mayores $d \epsilon+3^{\circ} \mathrm{C}$.

- La presencia de aguas calientes hia afectado considerablemente la producción fituplanctónica trayendo como consecuencia la escasa disponibilidad de alimento para los numerosos organismos que de ella se alimentan.

- La presericia de anomalias positivas con valores mayores de $+6.1{ }^{\circ} \mathrm{C}$ para el recurso macha ha desaparecido de en esta playa.

- En cuanto al crecimiento, se ha podido notar que durante el año de 1996 los especimenes experimentaron un crecimiento de $0.3 \mathrm{~mm}$ por mes, en cambio durante el año de 1997 este fue lento debido a la escasez de alimento en las aguas.

\section{REFERENCIASBIBLIOGRÁFICAS}

ARNTZ, W. y E. VALDIVIA 1985. "Incidencia del fenómeno del niño sobre los mariscos en el Litoral Peruano". Boletín. Volumen Extraordinario El niño su impacto en la fauna marina. Callao - Perú.

CASTELLANOS R. 1983. Evaluación de los recursos hidrobiológicos costeros (Mariscos) Tacna Vol. IV de la Dirección Regional de Pesquería de Tacna.
GOMEZ, J. 1982. Estudio Bioecológico de algunas especies de importancia comercial del litoral de Tacna. Tesis para obtar el Grado de Licenciado. Inst. Superior Pedagógico $102 \mathrm{pp}$.

IGREDA, M. y V. VLASKOVICH 1979. Estructura de la Población por talla y sexo de Mesodesma donacium en la playa de Asia del Departamento de Lima. 6to Congreso Nacional de Biología. Chiclayo - Perú. 
MEIER, H. 1973. Las Pesquerías de las Machas, Documenta $\mathrm{N}^{\circ} 30$ Ministerio de Pesquería.

MUÑANTE, L. 1980. Explotación de la Macha en el Litoral de Tacna. Revista Documenta $N^{\circ} 75$ Año VIII. Ministerio de Pesquería.

Fig. I. Carta de Posiciones.

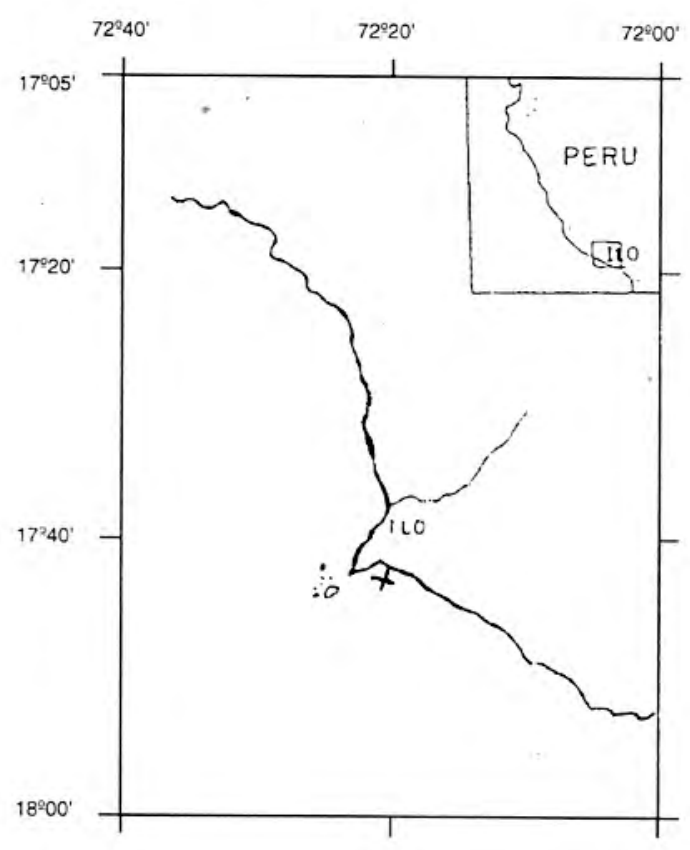

Fig. 2. Variación mensual de la Temperatura Superficial del Agua de Mar en la Playa Pozo de Lizas durante enero de 1995 a diciembre de 1997.

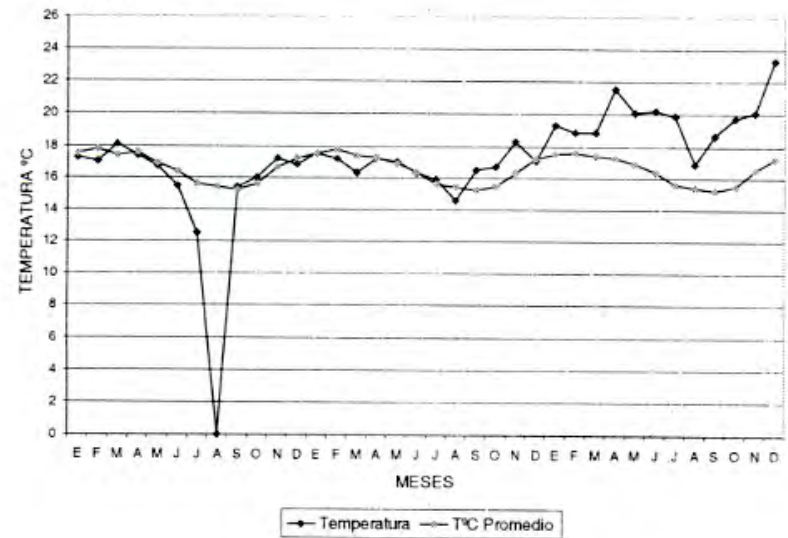

PONCE, E y A. SUPO 1979. Ventajas sanitarias y comerciales de la macha Liofilizadas Mesodesma donacium libro de Resumenes del 6to Congreso de Biología; 1er Simposium de Educación en Ciencias Biológicas Chiclayo - Perú.

TELLO, E. 1973. La Pesquería de la Macha. Revista Documenta $\mathrm{N}^{\circ}$ 28. Año III. Ministerio de Pesquería.

Fig. 3. Desviación mensual de la Temperatura Superficial del Agua de Mar en la Playa Pozo de Lizas, durante enero de 1995 a diciembre de 1997

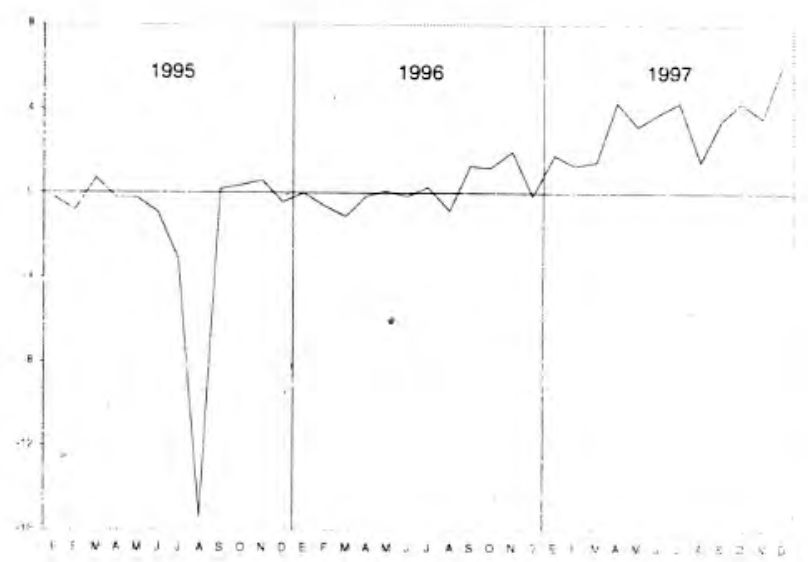

Fig. 4. Composición del Fitoplancton en la Playa Pozo de Lizas durante enero de 1995 a diciembre de 1997.

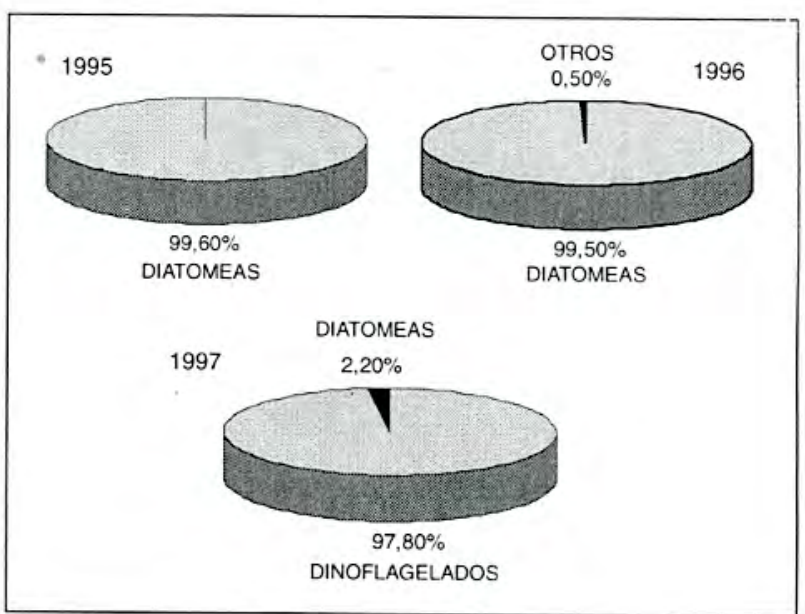


Fig. 5. Distribución de las Diatomeas en la Playa Pozo de Lizas durante enero de 1995 a diciembre de 1997.

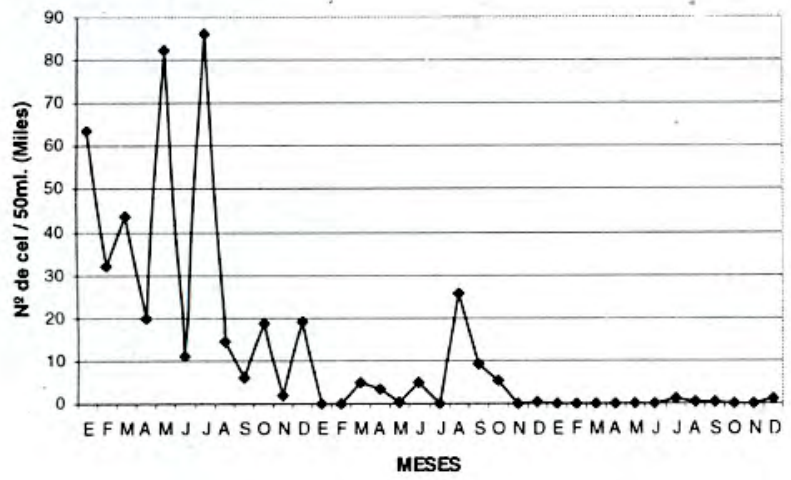

Fig. 6. Distribución de los Dinoflagelados en la Playa Pozo de Lizas durante enero de 1995 a diciembre de 1997.

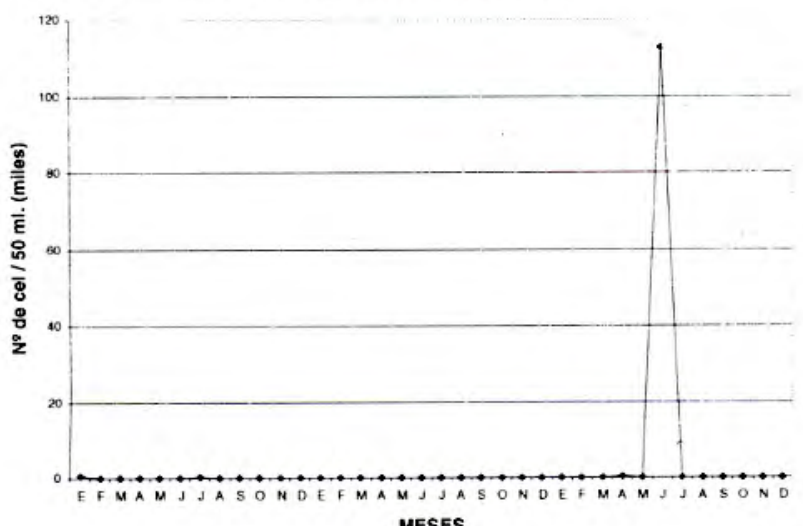

Fig. 8. Variación Estacional del Fitoplancton en la Playa Pozo de Lizas durante enero de 1995 a diciembre de 1997.

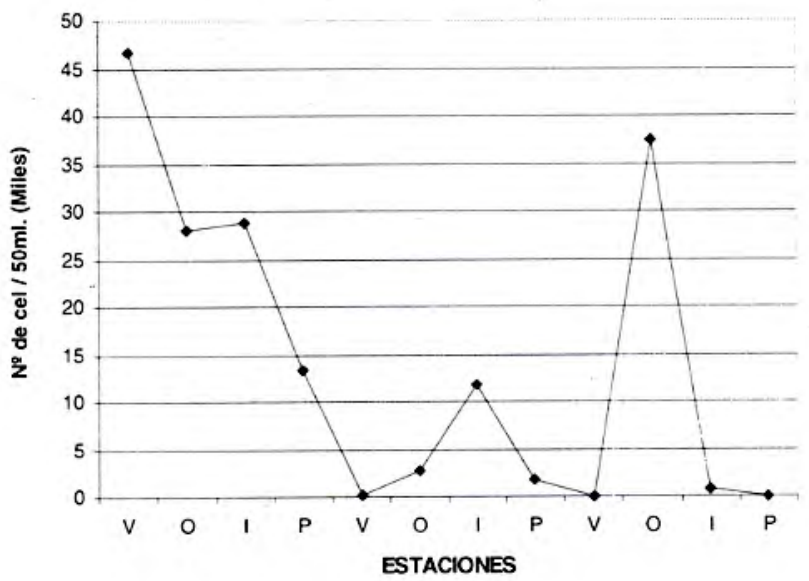

Fig. 9. Distribución del Indice de Diversidad en la Playa Pozo de Lizas durante enero de 1995 a diciembre de 1997.

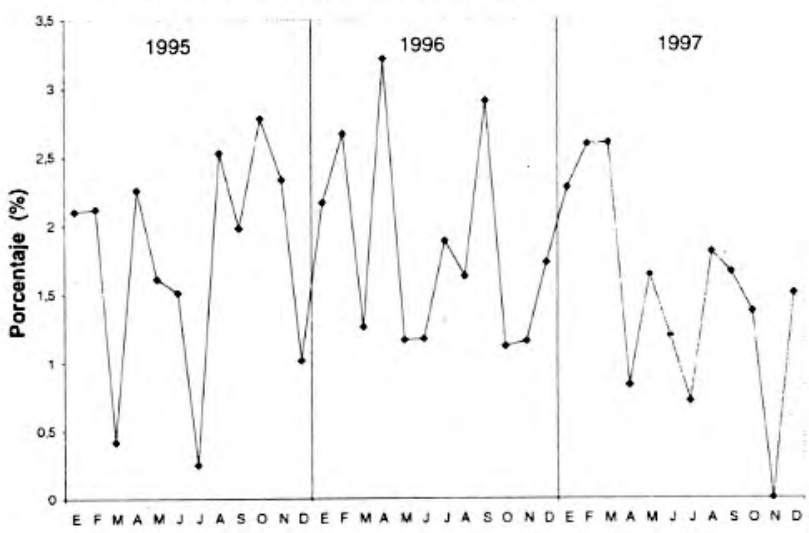

Fig. 7. Distribución de las Especies más Abundantes en la Playa Pozo de Lizas durante enero de 1995 a diciembre de 1997.

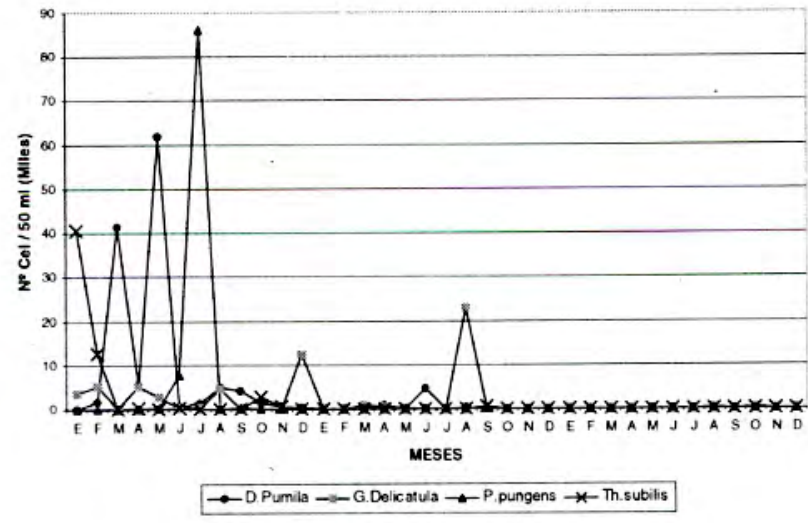

